\title{
High-speed detection at two micrometres with monolithic silicon photodiodes
}

Jason J. Ackert*1, David J. Thomson ${ }^{2}$, Li Shen², Anna C. Peacock², Paul E. Jessop ${ }^{3}$, Graham T. Reed², Goran Z. Mashanovich ${ }^{2}$ and Andrew P. Knights ${ }^{1}$

${ }^{1}$ Department of Engineering Physics, McMaster University, 1280 Main St. West, Hamilton ON, L8S 4L7 Canada

${ }^{2}$ Optoelectronics Research Centre, University of Southampton, Highfield, Southampton, Hampshire. SO17 1BJ, United Kingdom.

${ }^{3}$ Department of Physics and Computer Science, Wilfrid Laurier University, 75 University Ave. West, Waterloo, ON, N2L 3C5, Canada 


\section{Detector noise characterization}

Noise measurements have been performed on a $1 \mathrm{~mm}$ long photodetector. First, the signal-tonoise ratio (SNR) of the detected electrical signal was measured using an RF spectrum analyzer. The photodetector was probed with a semiconductor laser diode emitting at $1.96 \mu \mathrm{m}$ (EP-2000DM, Eblana Photonics) that carried a narrow-band signal (single RF tone). The signal was modulated on the optical carrier using an amplitude modulator (Photline MX2000-LN-10) driven by an RF signal generator (Agilent E8257D). By probing the detector with different signal frequencies $(1,2,5,8,10$ and $12.5 \mathrm{GHz}$ in our experiment), spectral characteristics of the SNR were obtained. As the detector noise was below the noise level of the RF spectrum analyzer, it was necessary to pre-amplify the detected signal using RF amplification (with bandwidth greater than $14 \mathrm{GHz}$ ) that provided gain of $60 \mathrm{~dB}$. Switching the laser ON and OFF did not change the observed noise level at the RF spectrum analyzer, suggesting the measurement was not limited by the laser noise characteristics (RIN - relative intensity noise). A block diagram of the experimental setup is shown in Fig. 1.

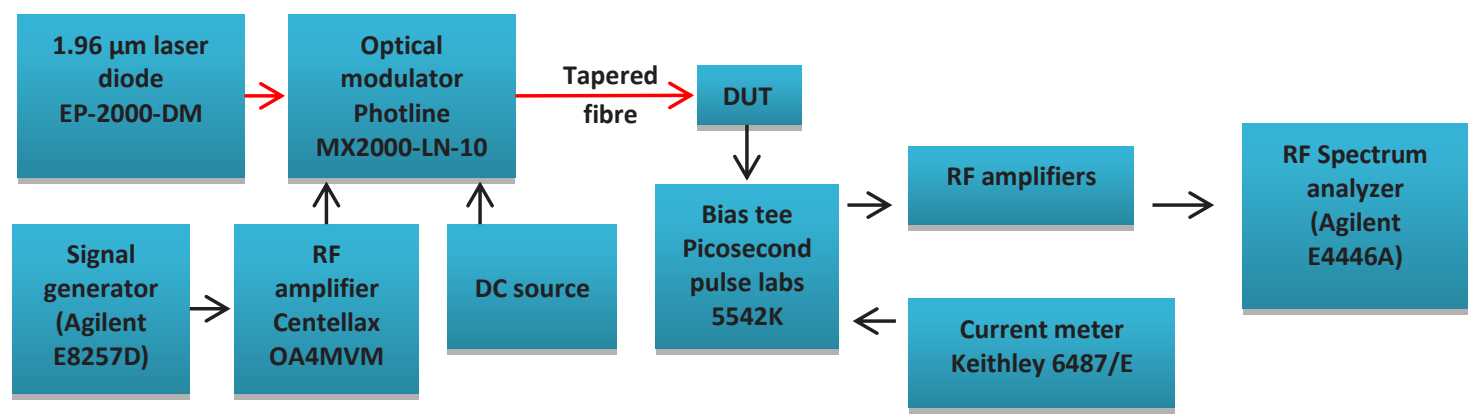

Figure 1 - Block diagram of the experimental configuration for noise measurements.

Noise-equivalent power (NEP) is defined as the quantity of incident light when the SNR=1. Thus:

$$
N E P=\frac{P}{S N R \Delta f},
$$

where $P$ is the incident power and $\Delta f$ is the measured noise bandwidth. The incident power was determined by measuring the optical power prior to the photodetector and subtracting the coupling loss, measured earlier to be $4 \mathrm{~dB}$. The noise level was measured with an instrument resolution bandwidth of $3 \mathrm{MHz}$. The measured values for the NEP obtained over the detector bandwidth are shown in Fig. 2 for bias voltages of -22 and $-25 \mathrm{~V}$. 


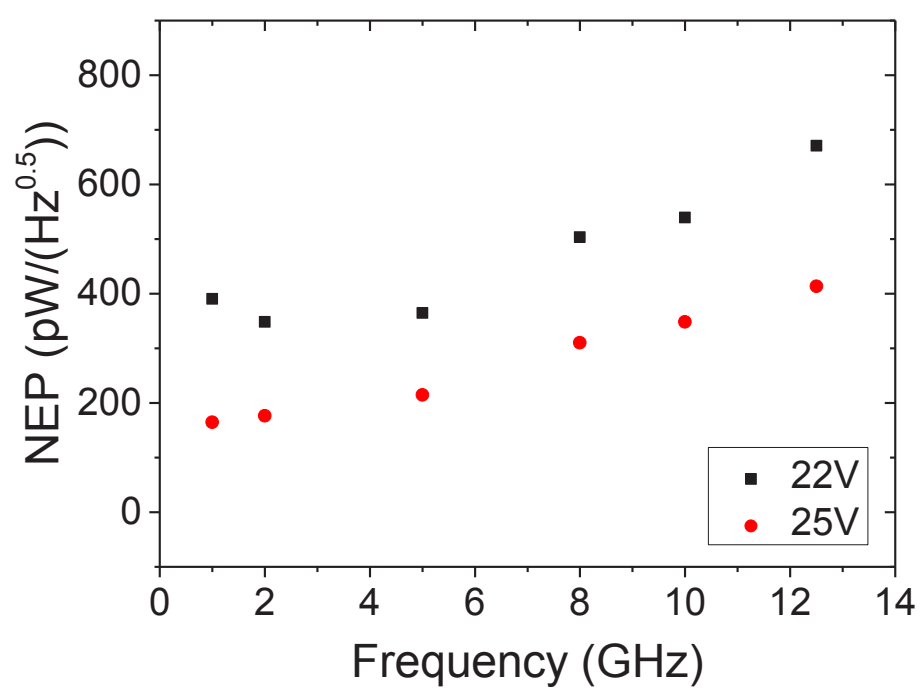

Figure 2 - NEP versus modulation frequency for reverse biases of $22 \mathrm{~V}$ and $25 \mathrm{~V}$.

The NEP can be related to the excess noise factor $^{1} F$ :

$$
N E P=\frac{\sqrt{2 q F(M, k) I_{d a r k}}}{R},
$$

where $q$ is the electron charge, $R$ is the unity-gain responsivity and $I_{\text {dark }}$ is the unity-gain dark current. The excess noise factor $F$ is a function of the multiplication factor $M$ and the ionization ratio $k$. The NEP is found to be lower at $25 \mathrm{~V}$ than $22 \mathrm{~V}$, a somewhat unexpected result. While the noise dependent bias characteristics of the device are intriguing, we reserve definitive explanation for future work and note that the significant result of this manuscript (mid-IR detection at high-speed) is unaltered. Further, the NEP measured for the device does not limit its use in network development.

[1] Gupta, M.C. and Ballato, J. The handbook of photonics. CRC press (2006). 


\section{Experimental setup for high-speed measurements}

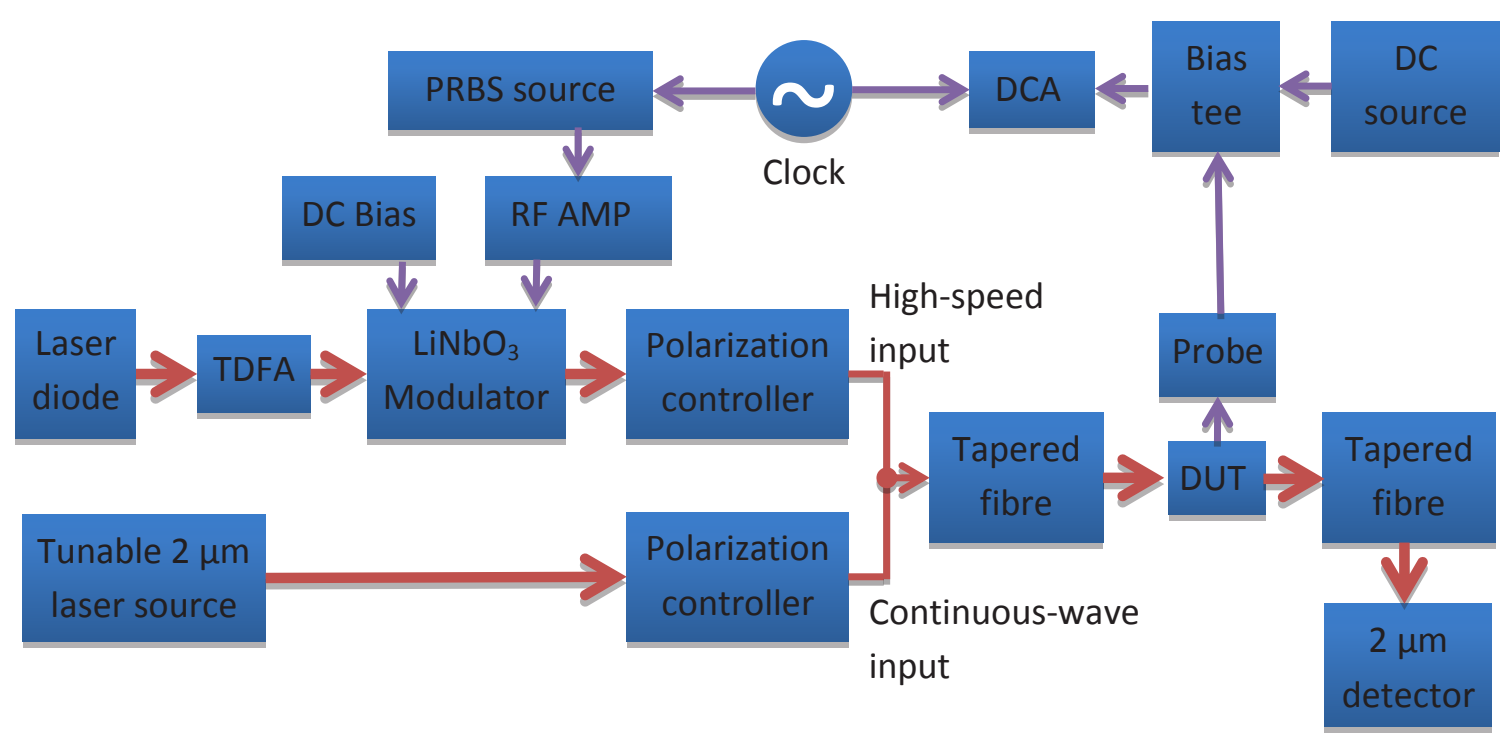

Figure 3 - A schematic diagram of the test setup. The high-speed and continuous-wave setup shared the same probe station but made use of a different light source. 